\title{
Cloning and Expression Analysis of Tomato SlSPS1 Gene
}

Dewen Zeng ${ }^{1,2}$, Yahui Liu ${ }^{2}$, Hui Zhang ${ }^{2}$, Xuedong Yang ${ }^{2}$, Longying Zhu ${ }^{2}$, Weimin Zhu ${ }^{1,2}$, Yingying Zhang ${ }^{2} \square$

1 College of Fisheries and Life Science, Shanghai Ocean University, Shanghai, 201306, China

2 Horticultural Research Institute, Shanghai Academy of Agricultural Sciences, Shanghai Key laboratory of Protected Horticulture Technology, Shanghai, 201403, China

$\square$ Corresponding author email: renlife@,163.com

Molecular Plant Breeding, 2022, Vol.13, No.2 doi: $\underline{10.5376 / \mathrm{mpb} .2022 .13 .0002}$

Received: 05 Jan., 2022

Accepted: 17 Jan., 2022

Published: 26 Jan., 2022

Copyright $\odot 2022$ Zeng et al., This article was first published in Molecular Plant Breeding in Chinese, and here was authorized to translate and publish the paper in English under the terms of Creative Commons Attribution License, which permits unrestricted use, distribution, and reproduction in any medium, provided the original work is properly cited.

Preferred citation for this article:

Zeng D.W., Liu Y.H., Zhang H., Yang X.D., Zhu L.Y., Zhu W.M., and Zhang Y.Y., 2022, Cloning and expression analysis of tomato SlSPS1 gene, Molecular Plant Breeding, 13(2): 1-11 (doi: 10.5376/mpb.2022.13.0002)

\begin{abstract}
Sucrose is the main product of plant photosynthesis and participates in many physiological and biochemical reactions of plants. Sucrose phosphate synthase is one of the key rate-limiting enzymes necessary for sucrose to enter various metabolic pathways, and its activity directly reflects the ability of sucrose synthesis in plants. In order to study the mechanism of sucrose synthesis in tomato, this study cloned a sucrose phosphate synthase gene from tomato pulp and named it SlSPS1. Through bioinformatics analysis, it was found that its cDNA is 3488bp in length, encoding 1054 amino acids, and there are 3 functional domain. There are 4 SPS genes in tomato, and their homology is relatively high. In Salicaceae crops, SISPS1 protein is closely related to potatoes, peppers and eggplants. The results of tissue-specific analysis found that SISPS1 was expressed in all tomato tissues, among which the expression was relatively high in the seedling stage and different development stages of the fruit. In order to further study the mechanism of SISPS1 in tomato growth and sucrose accumulation, an overexpression and knockout vector of SISPS1 was constructed and transformed into Micro-Tom to obtain transgenic positive lines. Analysis of the expression level of SISPS1 on the obtained transgenic lines showed that the expression levels of the overexpression lines at the seedling stage, fruit green ripe stage and ripening stage were significantly up-regulated compared with the wild type, and the enzyme activity and sucrose content increased. Compared with the wild type, the expression of SISPS1 at the seedling stage, green ripe stage and ripening stage of the knockout line was significantly down-regulated, and the enzyme activity and sucrose content were reduced. As for the mechanism of SISPS1 in tomato growth and fruit sucrose accumulation, functional verification is needed in the future.
\end{abstract}

Keywords Tomato; Clone; SISPS1; Expression; Analysis

Solanum lycopersicum belongs to Lycopersicon of Solanaceae, commonly known as tomato, and is a fruit and vegetable that is very popular among consumers. Carbohydrates affect the growth and development of fruit, and as signal molecules participate in regulating the expression of genes related to fruit ripening and the process of stress tolerance (Liao et al., 2020). The sugars in tomato fruit basically were glucose, fructose and sucrose. Sucrose is not only the main product of plant photosynthesis, but also participates in the physiological and biochemical processes of plants. There are three kinds of enzymes associated with sucrose metabolism in higher plants, sucrose phosphate synthase (SPS), sucrose synthase (SuSy), invertase (INV), among which, SPS is a ratelimiting enzyme and plays a certain role in sucrose metabolism. Its activity directly reflects the ability of sucrose synthesis in plants (Huber and Huber, 1996; Harbron, 1981).

At present, SPS genes have been successively cloned and studied in higher plants, such as Beta vulgaris, Cucumis melo, Manihot esculenta, Dendrobium officinale, Cerasus humilis, Mangifera indicaL, Dimocarpus longan and so on (Hao, 2012; Hou et al., 2008; Huang et al., 2018; Meng et al., 2013; Wang et al., 2017; Bai et al., 2019; Shuai et al., 2020). The current research results have found that SPS participates in plant growth, fruit ripening and other processes, and has the effect of responding to abiotic stress. However, at present, the transcriptional regulation process of SPS in plants, the molecular mechanisms and pathways involved in various biological functions are still unclear. Therefore, it is of great significance to analyze the function and regulation mechanism of SPS in sucrose metabolism and plant growth, which can provide a theoretical basis for increasing crop yield 
and improving crop quality.

In this study, SISPS1 gene was cloned from tomato fruit and analyzed by bioinformatics. Real-time quantitative polymerase chain reaction (qPCR) was used for tissue specificity analysis. In addition, overexpression and knockout vectors were constructed, and Micro-Tom was transformed by agrobacterium-mediated method. SlSPS1 expression analysis, SPS enzyme activity determination and sucrose determination were performed on the obtained transgenic lines.

\section{Results and Analysis}

\subsection{Cloning and bioinformatics analysis of SISPS1 gene}

Using tomato fruit genomic DNA and the whole gene cDNA obtained by reverse transcription as templates, primers were designed to clone the full length of SISPS1 gene and cDNA. The target fragment was detected by agarose gel electrophoresis. The size of cDNA was about $3488 \mathrm{bp}$, which was consistent with the expected results (Figure 1). Using ORF finder on NCBI, it was found that the CDS region of SISPS1 was $3165 \mathrm{bp}$, encoding 1 054 amino acids.

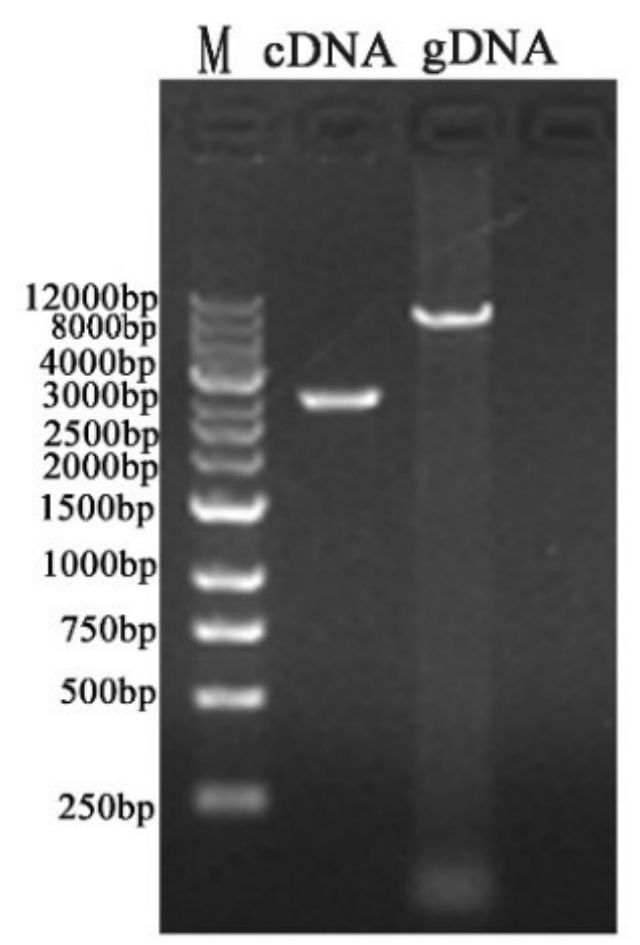

Figure 1 Full-length electrophoresis analysis of tomato SlSPS1 gene

Note: M: 1 kb DNA Ladder; g-DNA: Genomic DNA

Through the GENEBANK search of NCBI, it was found that there were four SPS with high protein homology in tomato, namely S1SPS1, SISPSB, SISPS4 and SISPSA2. Through the protein homologous sequence alignment analysis of these four genes (Figure 2), it was found that SISPS1 had high homology with the other three proteins, indicating that there may be some functional similarities among them. By predicting the domain of SISPS1 protein (Figure 3), it can be found that it had 3 domains, namely Sucrose_synth, Glycos_transf_1_4 and S6PP. The secondary structure of SISPS1 protein was predicted through the online website. The prediction results of the secondary structure of the protein were as follows: $\alpha$-helix accounted for $40.7 \%$, extended chain accounted for $13.76 \%$, irregular curl accounted for $38.99 \%$, and the tertiary structure of the protein was further constructed (Figure 4). The protein sequences of Solanaceae crops were obtained from NCBI and a phylogenetic tree was constructed (Figure 5). The results showed that SISPS1 was closely related to Solanum tuberosum, Capsicum annuum and Solanum melongena. 


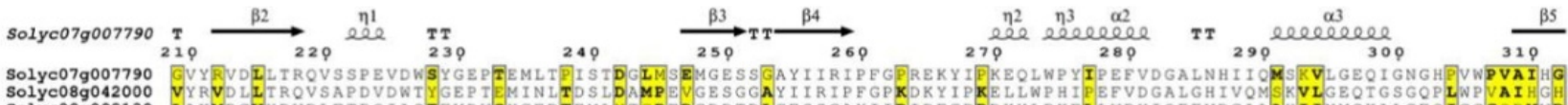

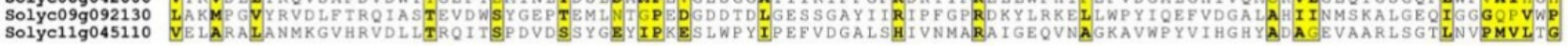

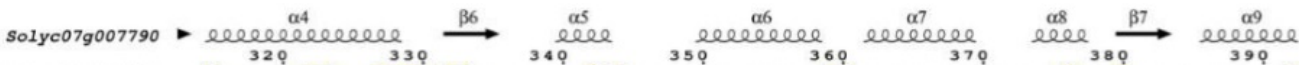

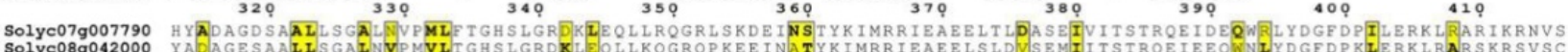
VPUL

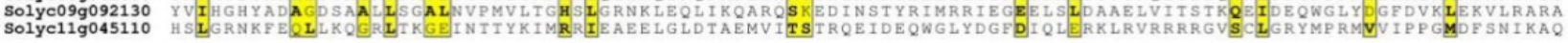

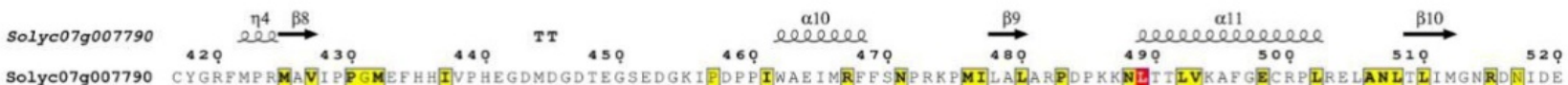

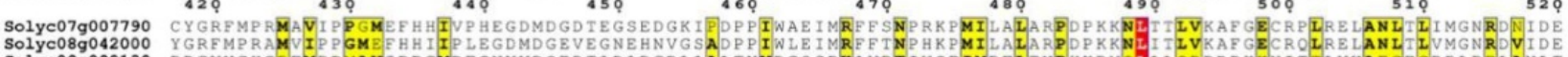

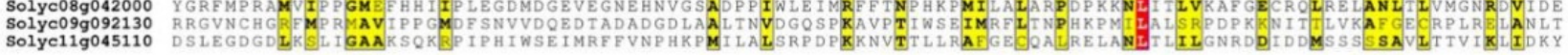

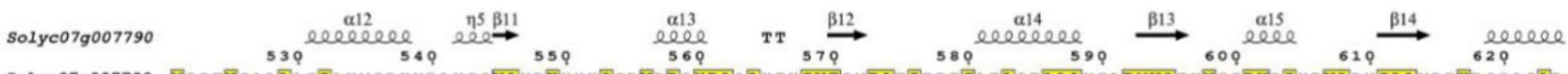

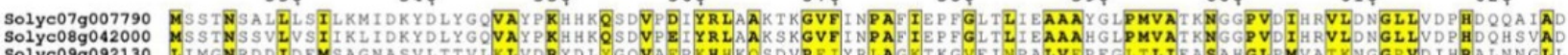

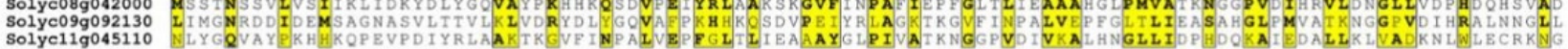

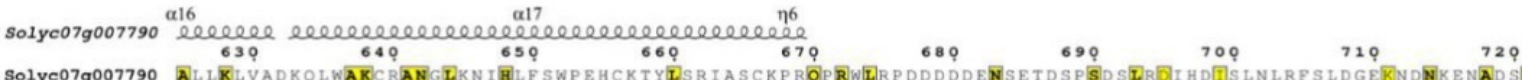

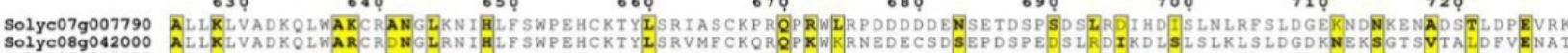

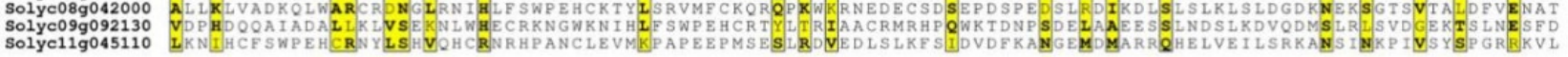

Solyc07g007790

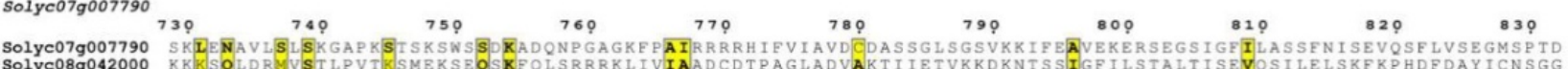

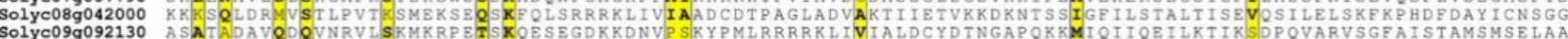

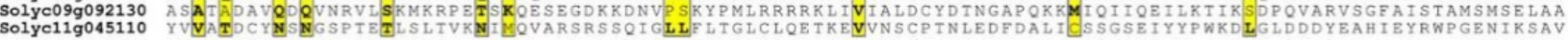

Solyc07g007790

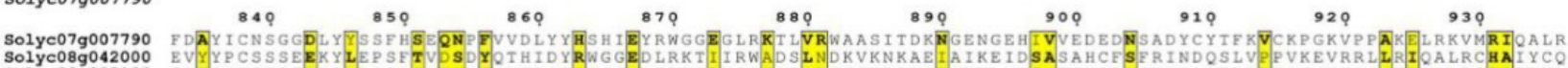

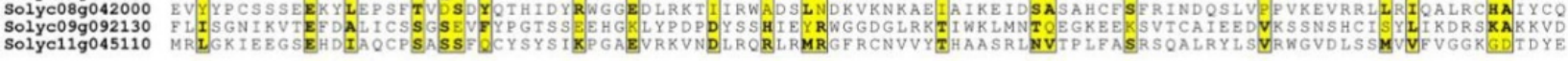

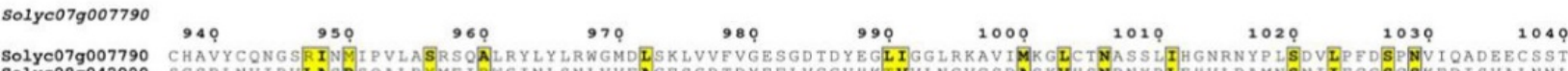

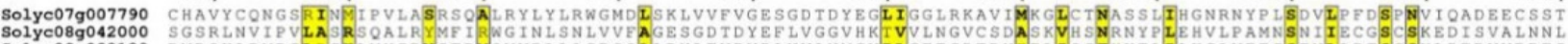

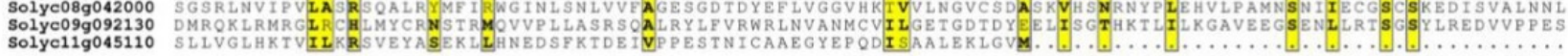

\begin{tabular}{|c|c|}
\hline Solyc07g007790 & 1050 \\
\hline $\begin{array}{l}\text { Solyc } \\
\text { Solye }\end{array}$ & $\begin{array}{l}\text { EIRSLLEKLAVLKG } \ldots \ldots \ldots \\
\text { GFSK }\end{array}$ \\
\hline $\begin{array}{l}\text { Solyc08gg042000 } \\
\text { Solyc09g092130 }\end{array}$ & PLIK IYTGGNETVEEYA NALKQVCR \\
\hline Solyc11g045110 & \\
\hline
\end{tabular}

Figure 2 Sequence alignment of SISPS1 and its homologous gene protein

Note: Solyc079007790: SISPS1 Solyc08g042000: SPSA2 Solyc09g092130: SPSB Solyc11g045110: SISPS4; Red box: Conservative sequences; Yellow box: More conservative sequences than white 
SISPS1

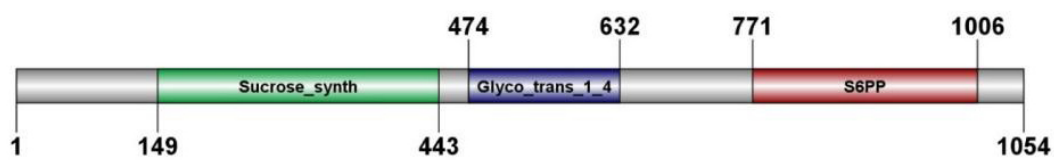

Figure 3 SISPS1 protein domain prediction

Note: Sucrose_synth: sucrose synthase; Glycos_transf_1_4: glycosyltransferase group 1; S6PP: sucrose-6-phosphohydrolase

A

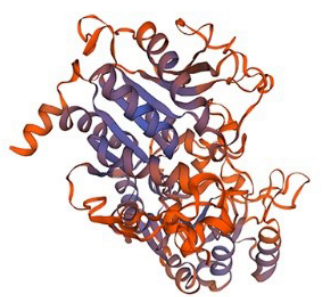

B

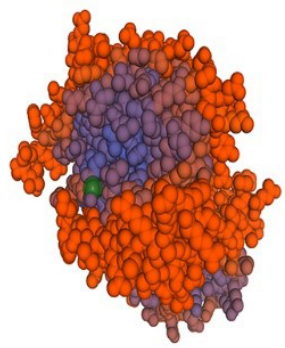

C

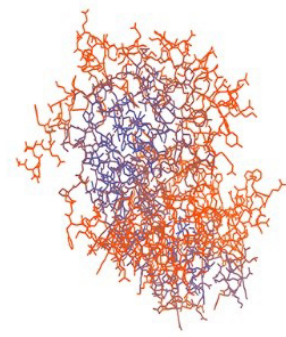

Figure 4 F Tertiary structure of SISPS1 protein

Note: A: Surface-shaped three-level structure diagram; B: Spherical three-level structure diagram; C: Linear three-level structure diagram

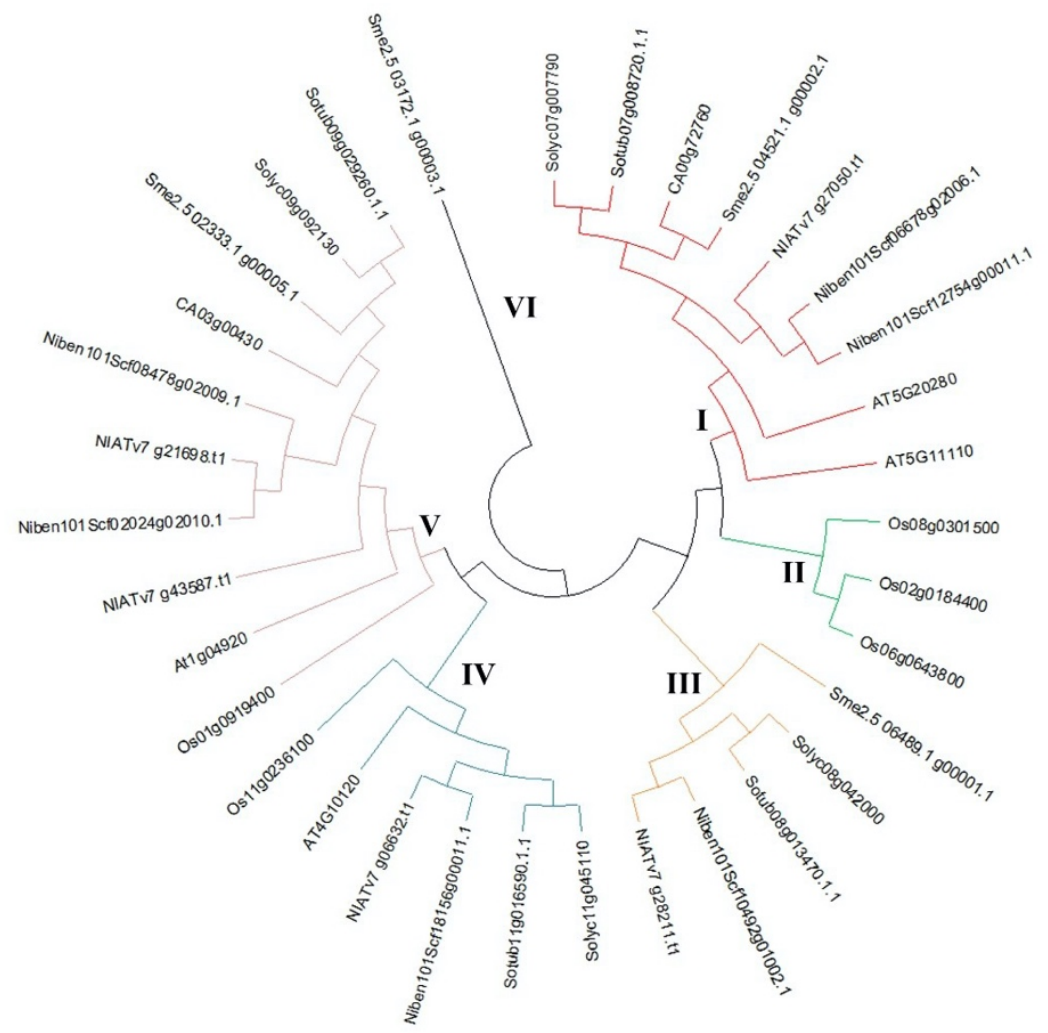

Figure 5 Protein evolutionary tree of SISPS1

\subsection{SISPS1 tissue specificity analysis}

After detecting the expression of SISPS1 in root, stem, leaf, seedling, flower and fruit (green ripe stage, slight maturity stage, half-ripe stage and ripening stage) of Micro-Tom, qPCR results showed that SISPS1 was expressed in all tomato tissues, and there were differences in expression patterns among different tissues, among which the expression was relatively high in the seedling stage and different development stages of the fruit (Figure 6). At 
the same time, the SPS enzyme activity in Micro-Tom tissues at different stages was measured. The results showed that the SPS enzyme activity was also relatively high in fruit and seedling, and the SPS enzyme activity was the highest in the half-ripe stage of fruit (Figure 7). It was speculated that SPS may play an important role in tomato fruit development and carbohydrate accumulation and metabolism.

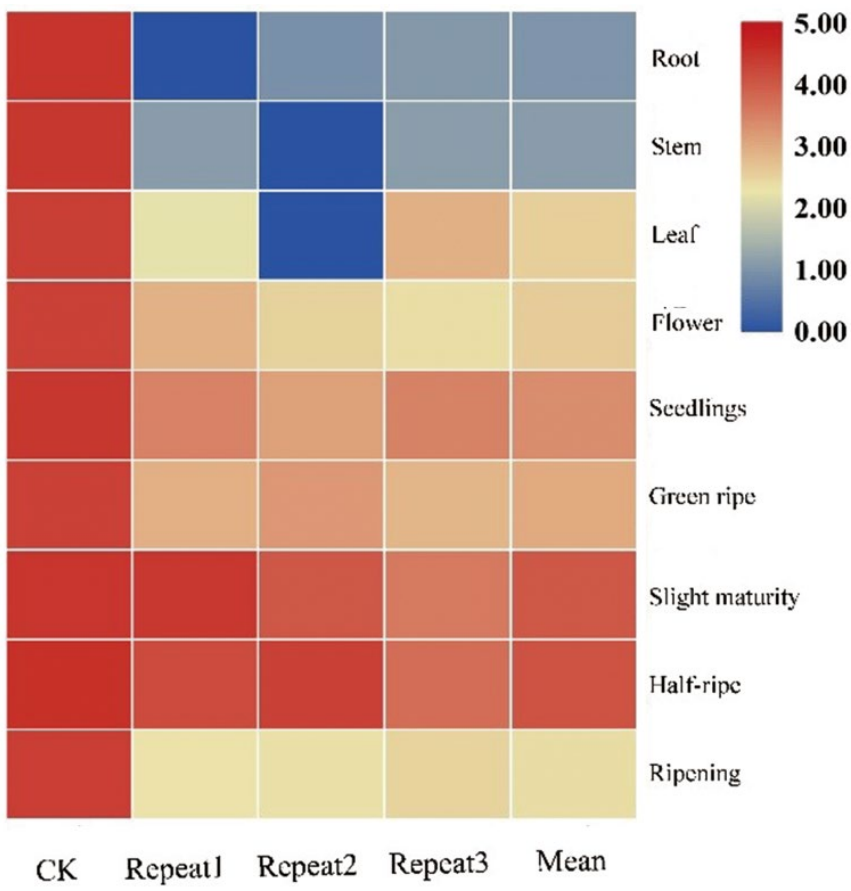

Figure 6 Expression analysis of SISPS1 in different organs of tomato model plant Micro-Tom

Note: Set the expression level of SISPS1 in the root to 1, use eIf as the internal reference gene, and set 3 repeats

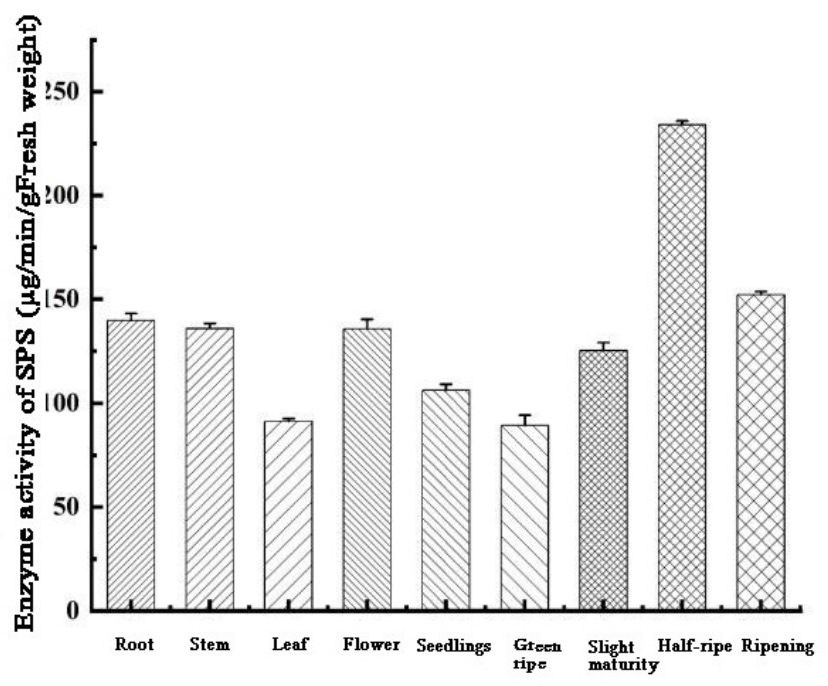

Figure 7 SPS enzyme activity analysis

\subsection{Construction and analysis of SISPS1 overexpression and knockout vector}

The SISPS1 gene was cloned and constructed by using pCAMBIA1300-pYAO-cas9 and pC1300-35S-4X Myc vectors, and the knockout vector and overexpression vector were constructed respectively. After sequencing correctly, it was transferred to Agrobacterium GV3101. The tomato model plant Micro-Tom was transformed by Agrobacterium mediated method, and the overexpression and knockout positive lines were obtained, which can be used for subsequent phenotypic analysis and the research of the mechanism of tomato growth and response to high temperature stress. 
After identifying the DNA level of the obtained SISPS1 overexpression transgenic lines, the expression of SISPSI in the transgenic lines was detected by qPCR. The results showed that the expression of SISPS1 at the seedling stage of the overexpression line was significantly up-regulated compared with the wild type. At the same time, the expression of SISPS1 at fruit green mature stage and mature stage of the overexpression line was also significantly up-regulated compared with the wild type (Figure 8). The SPS enzyme activities of the overexpression lines at seedling stage, green mature stage and red mature stage were measured. It was found that compared with the wild type, the enzyme activities of the overexpression lines increased significantly at seedling stage, green mature stage and red mature stage (Figure 9). The content of sucrose in the overexpression lines was further determined. The results showed that the content of sucrose at the seedling stage, green mature stage and red mature stage of the overexpression lines was significantly higher than that of the wild type (Figure 10).
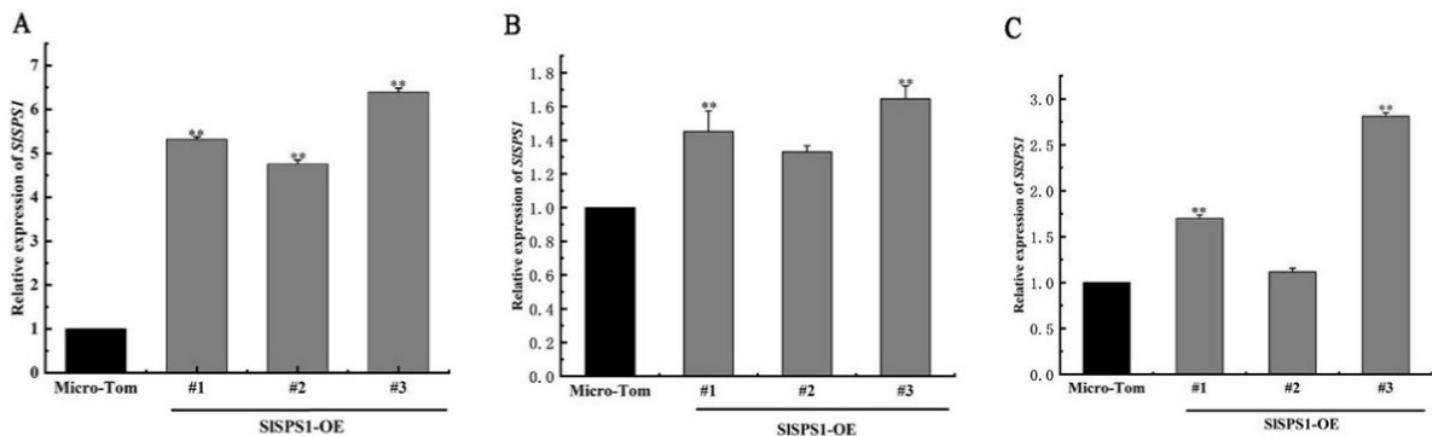

Figure 8 Analysis of expression level of overexpression line SISPS1

Note: A: Seedling period; B: Green mature period; C: Red mature period
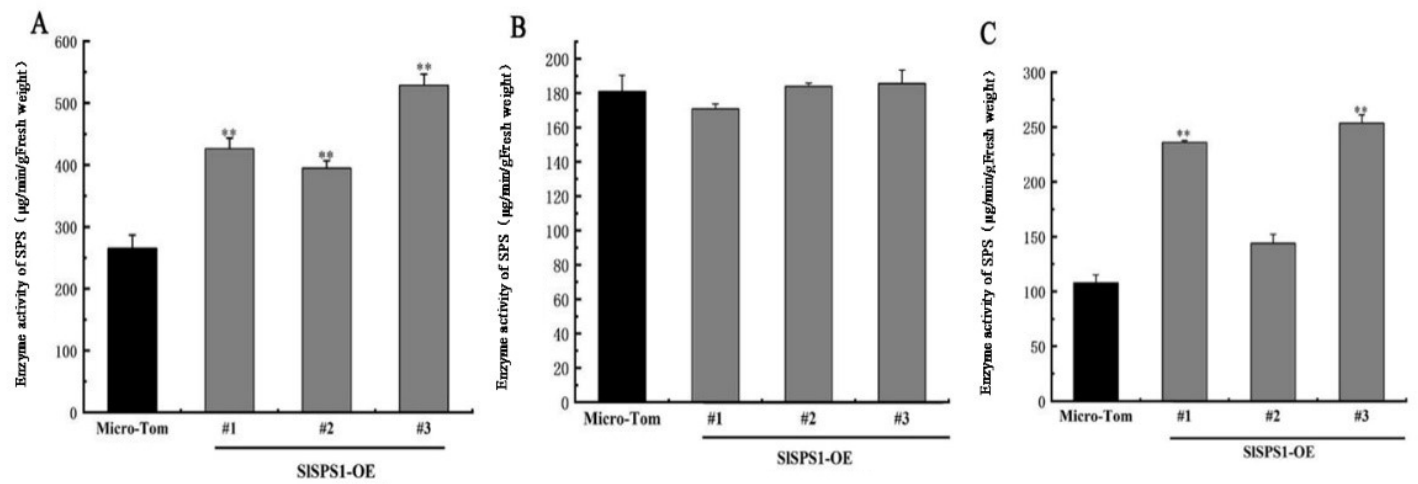

Figure 9 SPS enzyme activity of overexpression line

Note: A: Seedling period; B: Green mature period; C: Red mature period
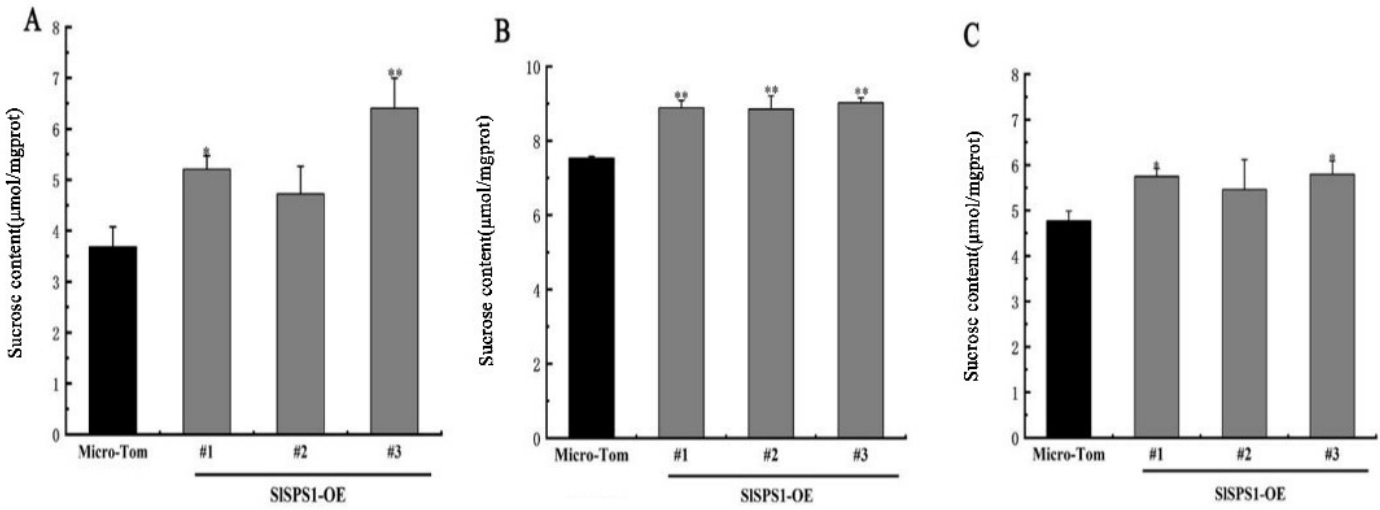

Figure 10 Over-expression plant sucrose content

Note: A: Seedling period; B: Green mature period; C: Red mature period 
Similarly, the DNA level of the knockout lines was identified and sequenced successfully. After obtaining the homozygous lines, the expression of SISPS1 in the transgenic lines was detected by qPCR. The results showed that the expression of SISPS1 at the seedling stage, green mature stage and red mature stage of the knockout line was significantly down-regulated compared with the wild type (Figure 11). The SPS enzyme activities of the knockout lines at seedling stage, green mature stage and red mature stage were measured. It was found that the enzyme activities of the knockout lines tended to decrease compared with the wild type (Figure 12). The content of sucrose in the knockout lines was measured. It was found that the content of sucrose at the seedling stage, green mature stage and red mature stage of the knockout lines was lower than that of the wild type (Figure 13).
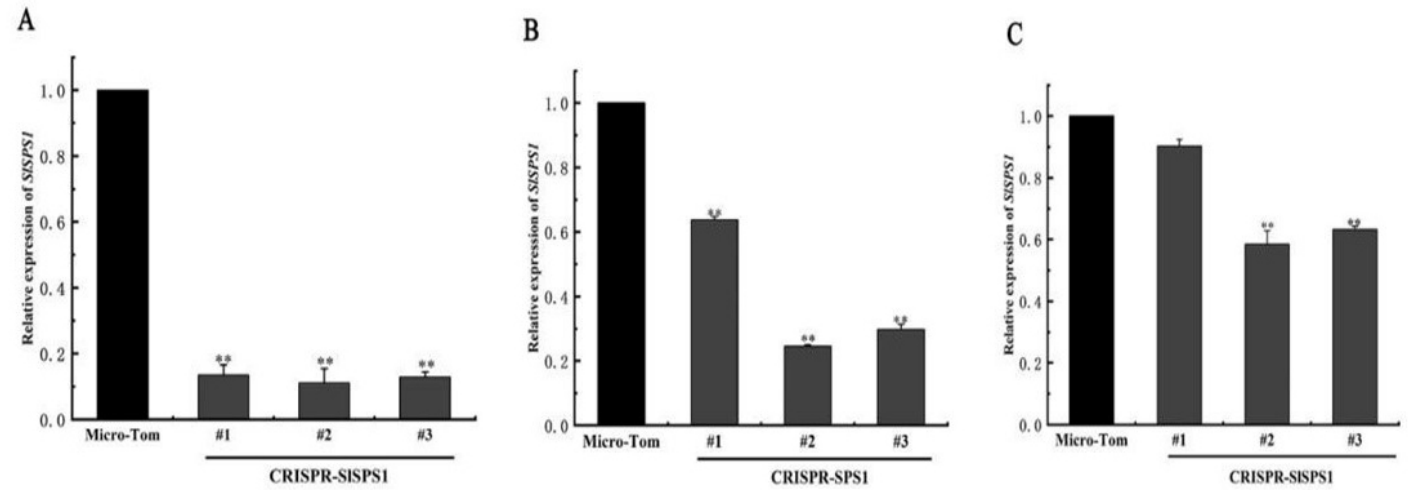

Figure 11 Sucrose content of overexpression line

Note: A: Seedling period; B: Green mature period; C: Red mature period
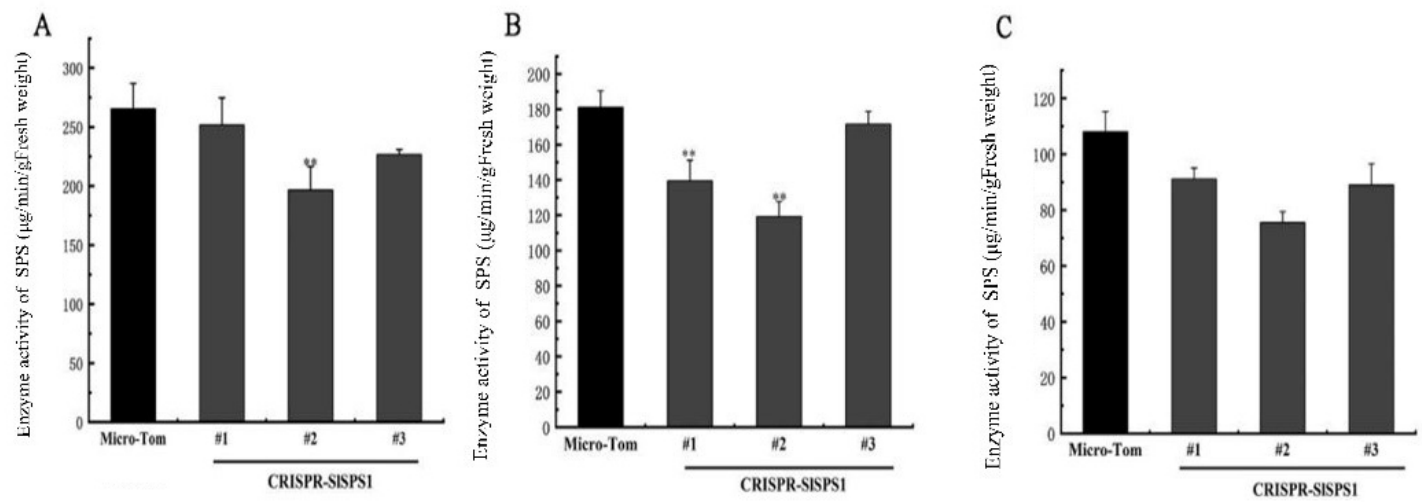

Figure 12 SPS enzyme activity of knockout line

Note: A: Seedling period; B: Green mature period; C: Red mature period
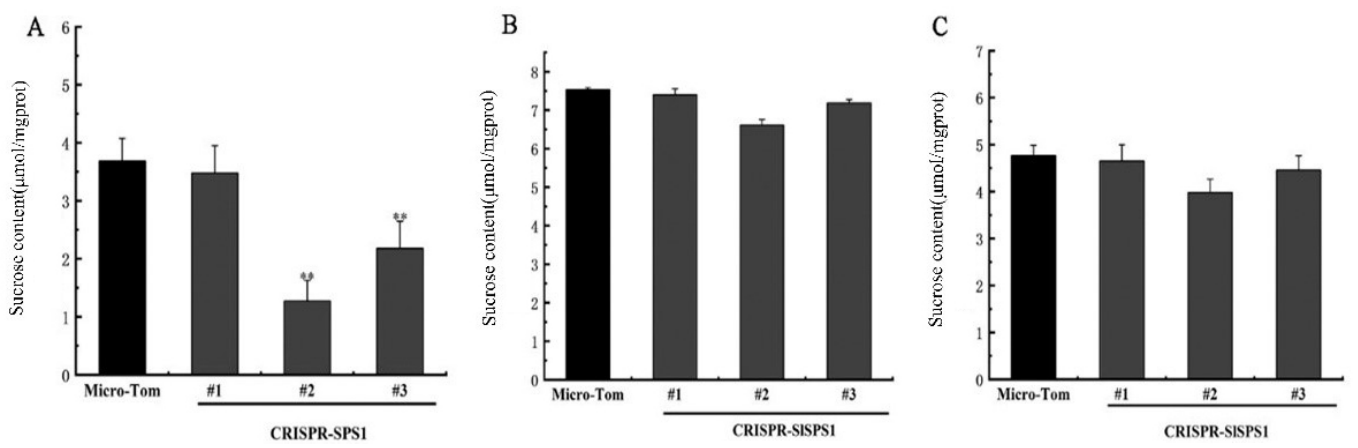

Figure 13 Knockout line SPS enzyme activity

Note: A: Seedling period; B: Green mature period; C: Red mature period 


\section{Discussion}

Sucrose is the main product of plant photosynthesis and plays an important role in plant growth, signal transduction and adaptation to environmental stress (Jiang et al., 2015). The activity of sucrose phosphate synthase directly reflects the ability of sucrose synthesis in plants. At present, SPS genes in higher plants have been cloned and studied. SPS gene was first found in Zea mays and Vicia faba (Hawker, 1971). Subsequently, it was found that SPS was involved in the regulation of carbon distribution in tomato leaves. When SPS activity increased in tomato leaves, sucrose increased and starch decreased (Worrell et al., 1991). During the development of tomato fruit, sucrose accumulation was related to the increase of SPS, and SPS would affect the sucrose accumulation process of tomato fruit (Dali et al., 1992). During the ripening process of Litchi chinensis, sucrose would accumulate in the aril. There are four SPS genes in Litchi chinensis, belonging to different SPS gene families, and their expression patterns were different. LcSPS1 and LcSPS4 were highly expressed in flowers, while LcSPS3 was highly expressed in mature leaves (Wang et al., 2018). In this study, it was also found that SISPS1 was expressed in tomato, and it was relatively high at seedling stage and different development stages of fruit. It was speculated that SISPS1 played a certain role in tomato fruit development and carbohydrate accumulation and metabolism. The expression of SISPS1 in the transgenic lines was analyzed. It was found that the expression levels of SISPSI in the overexpression lines at the seedling stage, fruit green ripe stage and ripening stage were significantly up-regulated compared with the wild type, and the enzyme activity and sucrose content increased. Previous studies have found that overexpression of Arabidopsis thaliana SPS gene would affect its growth and fiber development process, and the stem height of transformed plants increased significantly, which was related to internode elongation, stem diameter increase, fiber lengthening and biomass increase, and the SPS enzyme activity of transformed plants also increased (Park et al., 2008). Compared with the wild type, the expression of SISPS1 at the seedling stage, green ripe stage and ripening stage of the knockout line was significantly down-regulated, and the enzyme activity and sucrose content were reduced. Therefore, it could be speculated that SISPS1 gene played a certain role in tomato growth and sucrose accumulation.

Then the transgenic line was further applied to the study of sucrose accumulation, tomato growth and response to high temperature, so as to analyze the role of SISPS1 in tomato growth and fruit sucrose accumulation, so as to analyze the function and regulation mechanism of SPS in sucrose metabolism and plant growth.

\section{Materials and Methods}

\subsection{Experimental materials}

Tomato fruit was used as experimental material in this study. In addition, the root, stem, leaf, flower, seedling, green ripe stage, slight maturity stage, half-ripe stage and ripening stage of Micro-Tom in the same growth period were selected for tissue expression specificity analysis. After quick freezing with liquid nitrogen, the above samples were stored in an ultra-low temperature refrigerator at $-80^{\circ} \mathrm{C}$.

\subsection{Main reagents}

RNA extraction was used Biospin Plant Total RNA Extraction Kit (DNA-free) of Bioer Technology Co., Ltd. The reverse transcription kit was Hifair ${ }^{\mathbb{R}}$ II 1 st Strand cDNA Synthesis SuperMix for qPCR (gDNA digester plus) of Yeasen Biotech Co., Ltd. The fluorescent quantitative qPCR reagent was TB Green series of TaKaRa. Escherichia coli competent cells were purchased from Weidi Biotech Co., Ltd. Sucrose assay kit and SPS enzyme activity assay kit were purchased from Nanjing Jiancheng Bioengineering Institute.

\subsection{Main instruments and equipment}

Applied Biosystems Quantstudio5 real-time fluorescence quantitative PCR instrument (ABI company of the United States), micro nucleic acid protein analyzer (IMPLEN company), high-speed centrifuge (Eppendorf company of Germany), PCR instrument, thermostat water bath, enzyme-labeled instrument, ultra-clean bench, shaker, constant temperature culture chamber and autoclave. 


\subsection{RNA extraction and reverse transcription}

Preparation before RNA extraction: RNAase-free products were used during RNA extraction, which were dried after high-pressure steam sterilization. The RNA extraction process of tomato fruit referred to Biospin Plant Total RNA Extraction Kit (DNA-free) of Bioer Technology Co., Ltd., and after extraction, gel electrophoresis was used for examination., with total RNA as template, according to Hifair ${ }^{\circledR}$ II 1st Strand cDNA Synthesis SuperMix for qPCR (gDNA digester plus) kit of Yeasen Biotech Co., Ltd. for reverse transcription.

\subsection{Bioinformatics analysis}

The open reading frame of SISPS1 gene was found by using the tomato database Sol Genomics Network, and the homologous sequence of SISPS1 protein was searched by using the Blastp of NCBI. (https://prosite.expasy.org/) was used to predict the domain of SISPS1 protein, (https://www.predictprotein.org/) was used to predict the protein structure. Using the protein sequence of Solanaceae, the phylogenetic tree was drawn by MEGA-X software.

\subsection{Gene cloning}

Using tomato fruit cDNA as a template, primers were designed on both sides of the 5'UTR and 3'UTR of tomato SPS gene mRNA (Table 1) to clone the full length of the SISPS1 gene. The PCR reaction system was $25 \mu \mathrm{L}$, the ratio of each reactant should refer to the instructions of $\mathrm{I}-5^{\mathrm{TM}} 2 \mathrm{X}$ High-Fidelity Master Mix $25 \mu \mathrm{L}$. PCR reaction procedure was as follows: pre denaturation at $98^{\circ} \mathrm{C}$ for $5 \mathrm{~min}$; Denaturation at $98^{\circ} \mathrm{C}$ for $10 \mathrm{~s}$; Annealing at $58^{\circ} \mathrm{C}$ for $10 \mathrm{~s}$; Extension at $72^{\circ} \mathrm{C}$ for $30 \mathrm{~s}$, and for 10 cycles; Denaturation at $98^{\circ} \mathrm{C}$ for $10 \mathrm{~s}$; Annealing at $58^{\circ} \mathrm{C}$ for 10 $\mathrm{s}$; Extension at $72^{\circ} \mathrm{C}$ for $60 \mathrm{~s}$, and for 10 cycles; Denaturation at $98^{\circ} \mathrm{C}$ for $10 \mathrm{~s}$; Annealing at $58^{\circ} \mathrm{C}$ for $10 \mathrm{~s}$; Extension at $72^{\circ} \mathrm{C}$ for $90 \mathrm{~s}$, and for 10 cycles. After amplification, agarose gel electrophoresis was used to detect the amplified products. After the PCR products were cut and recovered, the target fragments were connected with the vectors, and then the connected products were transformed into E. coli competent cells. The colonies were selected for identification and sequencing, and the plasmids were extracted.

\subsection{The genetic transformation process of tomato}

Soak the seeds with sterile water overnight or $5 \mathrm{~h}$; sterilize with $20 \%$ sodium hypochlorite (12 \pm 2$)$ min; wash with sterile water for $4 \sim 6$ times, each time about $5 \mathrm{~min}$; sown on 1/2MS medium and cultivate in the dark for $2 \sim 3 \mathrm{~d}$. After exposure, transfer to $26 \sim 28^{\circ} \mathrm{C}$ light culture for about $5 \sim 7 \mathrm{~d}$, and the cotyledons began to unfold. When the true leaves did not appear and the cotyledons just emerged from the seed coat, the cotyledons were cut into leaf discs. Each cotyledon was cut into two pieces and connected to the nursing culture medium, about $60 \sim 80$ pieces each time. After $3 \mathrm{~d}$ of low light pre culture, the leaf discs would expand significantly. During the pre-culture period, the engineering bacteria should be prepared. It was best to draw a line $2 \mathrm{~d}$ in advance, and then pick a single bacteria and shake the bacteria under the dark condition of $26^{\circ} \mathrm{C}$ for about 16 hours to OD600 $=0.6 \sim 0.8$. Collect the bacterial solution at $5000 \mathrm{r} / \mathrm{min}$ for $8 \mathrm{~min}$, adjust OD $600=0.4$ with culture medium, $20 \mathrm{~mL}$ each time, and add $300 \mu \mathrm{mol} / \mathrm{L}(375 \mu \mathrm{mol} / \mathrm{L})$ AS. The prepared engineering bacterial solution should be used within $1 \mathrm{~h}$. Immerse the pre cultured leaf disc explants for $10 \mathrm{~min}$, suck them dry with sterile filter paper, place the leaf back upward on the nursing medium containing filter paper (do not pinch), and incubate in the dark at $26^{\circ} \mathrm{C}$ for $2 \mathrm{~d}$. Transfer to selective regeneration medium, culture under light with leaf back upward; Transfer once every $10 \mathrm{~d}$, wait for the regenerated buds to grow to about $1 \sim 2 \mathrm{~cm}$ (including the leaf part), cut off the albino explants (each bud should not be separated), and transfer the resistant buds into the fresh selective regeneration medium. Part of the non budding leaf discs would turn white, the cut ends would turn brown and there was no sign of healing. Such leaf discs should be removed in time. For leaf discs with the ability to grow regenerated buds, small callus would appear in the incision, and then buds would be differentiated. The emergence of buds would be in order. Pay attention to distinguish and mark them. When the resistant bud stem grew to $0.5 \sim 1 \mathrm{~cm}$, it was cut and placed in the rooting medium. When growing in the field, slowly open the bottle cap $3 \mathrm{~d}$ before transplanting, put it in the sterilized culture soil and wrap it in a transparent bag, uncover the culture after it grew, and finally transplant it to the field. 


\subsection{Real-time fluorescence quantitative PCR detection}

Using $e I F$ gene as an internal reference gene, real-time fluorescent quantitative PCR was used to detect the expression of SISPSI gene. The primers (Table 1), and the calculation method of relative expression amount were $2^{-\Delta \Delta \mathrm{CT}}$ method.

\subsection{SPS enzyme activity and sucrose determination}

The extraction and determination were carried out according to the method of sucrose phosphate synthase (SPS) determination kit and sucrose determination kit of Nanjing Jiancheng Bioengineering Institute.

Table 1 Primers and sequences used in the experiment

\begin{tabular}{lll}
\hline Primer name & Sequence & Usage \\
\hline SPS-ORF-F & GGAAACGATTGGATTAACAGTT & ORF amplification \\
SPS-ORF-R & TTATCCTTTGAGTACCGCTAGT & \\
SISPS-QF2 & CTGGTGGTCAGGTGAAGTATGTT & Fluorescence quantitative primer \\
SISPS-QR2 & TCTAGTAAGCAAGTCAACCCGATA & \\
eIF-F & ATCCTTCAGAGCGGTGTTCA & Internal reference primer \\
eIF-R & ATCTCAAGAGCCTCTGGTGG & \\
HYG-F & TCGTTATGTTTATCGGCACTTT & Transgenic resistance identification \\
HYG-R & GGGCGTCGGTTTCCACTAT & \\
CR-SPS-F & AGAATCTCTGTATTTTTAGC & Knockout material identification \\
CR-SPS-R & TACACAACGCATCAAAACAA & \\
SPS-OE-F & AACGCCGTCAAGAACGTGAG & Identification of overexpression materials \\
SPS-OE- R & CCTCGCAAGTTCAACAACATAC & \\
\hline
\end{tabular}

\subsection{Data processing}

Excel was used for data sorting, SPSS was used for data statistics and analysis, and Origin was used for drawing.

\section{Authors' contributions}

ZDW was the designer of the experiments and the executor of the research. ZDW and LYH completed the data analysis and wrote the first draft of the manuscript. ZH, YXD and ZLY participated in the experimental design and the analysis of experimental results. ZWM was the conceiver and the person in charge of the project. ZYY guided the experimental design, data analysis and paper revision. All authors read and approved the final manuscript.

\section{Acknowledgments}

This study was funded by the 'Establishment and Application of Molecular Markers for Tomato and Cabbage Quality Traits' Key Projects of Revitalizing Agriculture Through Science and Technology in Shanghai [Hu Nong Ke Gong Zi (2015) No. 6-1-7].

\section{References}

Bai B.B., Geng H.Y., Jing Y.L., Zhao Z.C., and Chen Y.Y., 2019, Cloning of mango MinSPS1 gene and construction of expression vector, Fenzi Zhiwu Yuzhong (Molecular Plant Breeding), 17(3): 855-861

Dali N., Michaud D., and Yelle S., 1992, Evidence for the involvement of sucrose phosphate synthase in the pathway of sugar accumulation in sucroseaccumulating tomato fruits, Plant Physiol., 99(2): 438-438

https://doi.org/10.1104/pp.99.2.434

PMid:16668903 PMCid:PMC1080480

Harbron S., Foyer C., and Walker D., 1981, The purification and properties of sucrose-phosphate synthase from spinach leaves: the involvement of this enzyme and fructose bio-phosphatase in the regulation of sucrose biosynthesis, Arch. Biochem. Biophys., 212(1): 237-246 https://doi.org/10.1016/0003-9861(81)90363-5

Hawker J.S., 1971, Enzymes concerned with sucrose synthesis and transformations in seeds of maize broad bean and castor bean, Photochemistry, 10(10): $2313-2322$ https://doi.org/10.1016/S0031-9422(00)89872-6

Hao H., 2012, Cloning and genetic transformation of sugar beet sucrose phosphate synthase (SPS) gene, Thesis for M.S., Heilongjiang University, Supervisor: Ma L.B., pp.26 
Hou L.X., He Q.W., Zhao S.Y., Jiao Z.G., Wang C.Q., and Dong Y.M., 2008, Gene cloning of melon sucrose phosphate synthase and construction of plant engineering vector, Guoshu Xuebao (Journal of Fruit Trees), 25(4): 548-551

Huber S.C., and Huber J.L., 1996, Role and regulation of sucrose phosphate synthase in higher plants, Plant Physiol., 47(1): 431-444. https://doi.org/10.1146/annurev.arplant.47.1.431 PMid:15012296

Huang T.W., Luo X.L., Shan Z.Y., and Zhu Y.M., 2016, Cloning and tissue expressions of sucrose phosphate synthase gene in cassava, Fujian Nongye Xuebao (Fujian Journal of Agriculture Sciences), 31(12): 1273-1279

Jiang S.Y., Chi Y.H., Wang J.Z., Zhou J.X., Cheng Y.S., Zhang B.L., and Ali Ma1., 2015, Sucrose metabolism gene families and their biological functions, Sci. Rep., 30(5): 1758-1782.

https://doi.org/10.1038/srep17583

PMid:26616172 PMCid:PMC4663468

Liao G.L., Zhong M., Huang C.H., Jia D.F., and Xu X.B., 2020, Research progress in fruit sugar metabolism and related enzyme gene, Jiangxi Nongye Daxue Xuebao (Journal of Jiangxi Agricultural University), 42(1): 187-195

Meng H.L., Yang S.C., Zha Y.H., and Wen G.S., 2013, Cloning and prokaryotic expression of sucrose phosphate synthase gene from Dendrobium candidum, Xibei Zhiwu Xuebao (Acta Botanica Boreali-Occidentalia Sinica), 33(4): 692-696

Park J.Y., Canam T., Kang K.Y., Ellis D.D., and Mansfield S.D., 2008, Over-expression of an arabidopsis family A sucrose phosphate synthase (SPS) gene alters plant growth and fibre development,Transgenic Res, 17: 181-192 https://doi.org/10.1007/s11248-007-9090-2 PMid:17415671

Shuai L., Fei Y.L., Liao L.L., Liu Y.F., Duan Z.H., and Song Z.H., 2020, Longan SPS gene cloning and expression analysis, Nanfang Nongye Xuebao (Southern Agricultural Journal), 51(7): 1529-1536

Wang J., Du J., Mu X., and Wang P.F., 2017, Cloning and characterization of the Cerasus humilis sucrose phosphate synthase gene (ChSPS1), PLoS One, 12 (10): e0186650 https://doi.org/10.1371/journal.pone.0186650 PMid:29036229 PMCid:PMC5643142

Worrell A.C., Bruneau J.M., Summerfelt K., Boersig K., and Voelker T.A., 1991, Expression of a maize sucrose phosphate synthase in tomato alters leaf carbohydrate partitioning, The Plant Cell., 3: 1121-1130 https://doi.org/10.1105/tpc.3.10.1121 PMid:1840396 PMCid:PMC160077

Wang D., Zhao J., Hu B., Li J.Q., Chen L.H., Qin Y.H., and Hu G.B.,2018, Identification and expression profile analysis of the sucrose phosphate synthase gene family in Litchi chinensis Sonn., Peer J., 6(3): e4379

https://doi.org/10.7717/peerj.4379 PMid:29473005 PMCid:PMC5816967 